\title{
Einkommensverhältnisse der freien Ärzteschaft der Schweiz in den Jahren 2001 und 2002
}

\section{Niklaus Hasler}

\begin{abstract}
Gutachten erstelllt im Auftrage der Verbindung der Schweizer Ärztinnen und Ärzte FMH. Das gesamte Gutachten mit 59 Seiten, 27 Tabellen und 28 Graphiken kann im Internet über die Homepage der FMH www.fmh.ch über $\rightarrow$ Deutsch $\rightarrow$ Über uns $\rightarrow$ Ärzteeinkommen abgerufen werden. Die Numerierung der Tabellen und Graphiken bezieht sich auf diesen vollständigen Bericht.
\end{abstract}

Korrespondenz:

Dr. med. Niklaus Hasler

Huzlenstrasse 3

8604 Volketswil

nik@hin.ch

\section{A. Einleitung}

Mit Datum vom 28. Januar 2005 hat die Verbindung der Schweizer Ärztinnen und Ärzte den Auftrag erteilt, die Einkommensverhältnisse der freien Ärzteschaft der Schweiz für das Einkommensjahr 2002 erneut durch eine Vollerhebung zu analysieren.

\section{A.1.1. Bemerkung zu TarMed}

Der grundsätzlich neue, gesamtschweizerisch gültige Arzttarif wurde für die Behandlungen nach Unfallversicherungsgesetz, nach Invalidenversicherung und nach Militärversicherungsgesetz auf den 1. Mai 2003 (geschätztes Honorarvolumen für das Jahr 2003 rund 5,3\% des gesamten Bruttohonorarvolumens) und zusätzlich für alle Behandlungen nach Krankenversicherungsgesetz auf den 1. Januar 2004 eingeführt (damit 100\% des Bruttohonorarvolumens gemäss TarMed-Tarif abgerechnet).

Das vorliegende Einkommensgutachten der Ärzteeinkommen des Jahres 2002 ist somit das letzte Gutachten, das vollständig ohne Anwendung des TarMed-Tarifs erhoben wird.

Das nächste Gutachten der Einkommen des Jahres 2003 (erscheint in der zweiten Hälfte 2006) umfasst schon einen kleinen Teil nach TarMed-Tarif abgerechneter Behandlungen (rund 5,3\% des Bruttohonorarvolumens). Das übernächste Gutachten der Einkommen des Jahres 2004 (erscheint in der zweiten Hälfte des Jahres 2007) umfasst nur noch Bruttoeinkünfte, die alle nach TarMed-Tarif abgerechnet worden sind.

Die Erhebung schliesst direkt an die früheren 20 Berichte an, welche von Dr. Niklaus Hasler zwischen August 1977 und Juli 2004 erstellt wurden.

Hier folgt eine Zusammenfassung der wichtigsten Resultate, Tabellen und Graphiken. In dieser Zusammenfassung werden bewusst nur die Zahlen für die Ärzte unter 66 Jahren angegeben. In der Vollversion sind jeweils auch die Zahlen für die Ärzte über 65 Jahre und die Zahlen für alle Ärzte (unabhängig vom Alter) angegeben.

Zudem werden in der Vollversion noch weitere Kriterien genau untersucht, wie z.B. die Abhängigkeit des Einkommens von der Personal- lohnsumme, vom Alter des Arztes oder von der Zeit in Jahren seit der Praxiseröffnung.

Im nächsten Kapitel wird die wichtigste Änderung der Erhebung beschrieben, welche in den Zeitraum 1997/98 bis 2001 fällt, nämlich der Übergang von der Vergangenheitsbesteuerung auf die Gegenwartsbesteuerung.

\section{A.2. Übergang auf die}

\section{Gegenwartsbesteuerung:}

Im Rahmen der eidgenössischen Steuerharmonisierung wurde auch die Veranlagung der AHVBeiträge der Selbständigerwerbenden von der Vergangenheitsbesteuerung auf die Gegenwartsbesteuerung umgestellt.

Das Durchschnittseinkommen der Jahre 1997 und 1998 (30. Wehrsteuerperiode) ist die Grundlage für die Verfügung der in den Jahren 1999 und $2000 \mathrm{zu}$ bezahlenden persönlichen AHVBeiträge.

Die Berufseinkommen der Jahre 1999 und 2000 fallen in die Bemessungslücke und können deshalb nicht erhoben und analysiert werden.

Ab dem Jahr 2001 gilt die Gegenwartsbesteuerung, d.h. das Einkommensjahr 2001 entspricht auch dem AHV-Beitragsjahr 2001.

Bis zu den Einkommen der 30. Wehrsteuerperiode (Einkommensjahre 1997/98) konnten die Ärzteeinkommen nur als zweijähriger Mittelwert im Zweijahresrhythmus erhoben werden.

Ab dem Jahr 2001 können die Ärzteeinkommen aufgrund der verfügten persönlichen AHVBeiträge als Jahreseinkommen im Einjahresrhythmus erhoben werden.

\section{A.3. Probleme mit dem Übergang}

Der föderalistische Aufbau der Schweizerischen Eidgenossenschaft bedingt, dass einzelne Gesetze nicht zum gleichen Zeitpunkt in allen Kantonen der Schweiz realisiert werden. Aus diesem Grunde gab es beim Einkommensgutachten vom Juli 2004 in den Kantonen Tessin, Wallis und Waadt einige Schwierigkeiten.

Heute kann jedoch eindeutig festgestellt werden, dass alle Kantone den Übergang zur Gegenwartsbesteuerung zur Bemessung der per- 
sönlichen AHV-Beiträge vollständig eingeführt haben.

\section{Resultate}

\section{D.1. Allgemeines}

Zur Überprüfung der im Juli 2004 publizierten Einkommenszahlen 2001 wurden diese Werte in der vorliegenden Erhebung erneut erhoben und analysiert. Die revidierten, jetzt publizierten Werte liegen durchwegs leicht tiefer, aber doch recht nahe an den Werten der Erhebung vom Juli 2004:

- Für alle Ärzte unabhängig vom Alter: (Mittelwert des AHV-pflichtigen Einkommens 203855 statt $205466=-0,78 \%$; Zentralwert des AHV-pflichtigen Einkommens 164700 statt $165200=-0,30 \%)$.

- Für alle Ärzte unter 66 Jahren: (Mittelwert des AHV-pflichtigen Einkommens 210723 statt $212494=-0,83 \%$; Zentralwert des AHVpflichtigen Einkommens 171500 statt 172200 $=-0,41 \%)$.

- Für alle Ärzte über 65 Jahre: (Mittelwert des AHV-pflichtigen Einkommens 92081 statt $94152=-2,20 \%$; Zentralwert des AHV-pflichtigen Einkommens 45100 statt $40800=$ $+10,54 \%)$.

Für die einzelnen Spezialitäten sind die Unterschiede äusserst uneinheitlich und unterschiedlich. Hier einige Beispiele:

- Für die Gynäkologen unter 66 Jahren (Mittelwert des AHV-pflichtigen Einkommens 249477 statt $253434=-1,56 \%$, Zentralwert des AHV-pflichtigen Einkommens 219100 statt $220500=-0,63 \%)$.

- Für die Urologen unter 66 Jahren (Mittelwert des AHV-pflichtigen Einkommens 312410 statt $329133=-5,08 \%$, Zentralwert des AHV-pflichtigen Einkommens 256900 statt $270800=-5,13 \%)$.

- Für die Psychiater unter 66 Jahren (Mittelwert des AHV-pflichtigen Einkommens 117513 statt $117389=+0,11 \%$, Zentralwert des AHV-pflichtigen Einkommens 107500 statt $110100=-2,36 \%$ ).

- Für die Allgemeinpraktiker unter 66 Jahren (Mittelwert des AHV-pflichtigen Einkommens 194738 statt $198139=-1,72 \%$, Zentralwert des AHV-pflichtigen Einkommens 178000 statt $181600=-1,98 \%)$.

Genau so wie die absoluten Werte für die Einkommen 2001 müssen die Veränderungen der Einkommen zwischen 1997/98 und 2001 wie untenstehend angegeben korrigiert werden.

\section{Einkommensentwicklung 1997/98}

bis 2001

Vergleich Gutachten Juli 2004 zu Gutachten Oktober 2005.

Teuerung 1997/98 bis $2001+3,40 \%$.

Veränderung des mittleren AHV-pflichtigen Ärzteeinkommens 1997/98 bis 2001:

- Alle Ärzte unabhängig vom Alter: $+2,53 \%$ statt $+3,37 \%$;

- Ärzte unter 66 Jahren: $+2,98 \%$ statt $+3,85 \%$;

- Ärzte über 65 Jahre: $+7,88 \%$ statt $+10,30 \%$.

Veränderung des zentralen AHV-pflichtigen Ärzteeinkommens 1997/98 bis 2001:

- Alle Ärzte unabhängig vom Alter: +0,67\% statt $+0,98 \%$;

- Ärzte unter 66 Jahren: $+1.18 \%$ statt $+1,59 \%$;

- Ärzte über 65 Jahre: -13,77\% statt -21,99\%.

Einkommensentwicklung 2001 bis 2002

Der Anstieg der Teuerung zwischen 1997/98 und 2001 betrug $+0,63 \%$. Im gleichen Zeitraum ist das mittlere Ärzteeinkommen (AHV-pflichtiges Berufseinkommen) für folgende Teilgesamtheiten der freien Ärzteschaft der Schweiz wie folgt angestiegen:

- alle Ärzte von 203805 auf 207752 um +1,94\%;

- Ärzte unter 66 Jahren von 210723 auf 215071 um $+2,06 \%$;

- Ärzte über 65 Jahre von 92081 auf 91889 um $-0,21 \%$.

Das zentrale Ärzteeinkommen (das Einkommen der einen Hälfte der angegebenen Ärzte liegt unter, das Einkommen der anderen Hälfte der angegebenen Ärzte liegt über dem zentralen Ärzteeinkommen) ist im gleichen Zeitraum für die gleichen Teilgesamtheiten wie folgt angestiegen: - alle Ärzte von 164700 auf 167900 um +1,94\%; - Ärzte unter 66 Jahren von 171500 auf 173800 um $+1,34 \%$;

- Ärzte über 65 Jahre von 45100 auf 48000 um $+6,43 \%$.

Mit Ausnahme der Ärzte über 65 Jahre hat sich das mittlere Ärzteeinkommen zwischen 2001 und 2002 minim stärker wie die Teuerung entwickelt. Es ist ein reales Ansteigen von 1,31\% bis $1,43 \%$ festzustellen. Beim zentralen Ärzteeinkommen hingegen ist ein reales Ansteigen von $0,71 \%$ bis $1,31 \% \mathrm{zu}$ verzeichnen.

Bei den Ärzten über 65 Jahre ist beim mittleren Ärzteeinkommen ein reales Absinken von $-0,84 \%$ und beim zentralen Ärzteeinkommen ein realer Zuwachs von $+5,80 \%$ festzustellen.

Wenn die Zahlen zusammen mit den weiteren definitiv verfügten AHV-Einkommen in ein 
Tabelle 23a

Indexzahlen des AHV-pflichtigen Einkommens der freien Ärzteschaft der Schweiz (bis 1975/76 unter 63 Jahren, ab $1977 / 78$ unter 66 Jahren). $1971 / 72$ bis 2002: Erster Teil 1971/72 bis 1983/84. Alle Indexreihen sind auf der Basis 1971/72=100,0 berechnet.

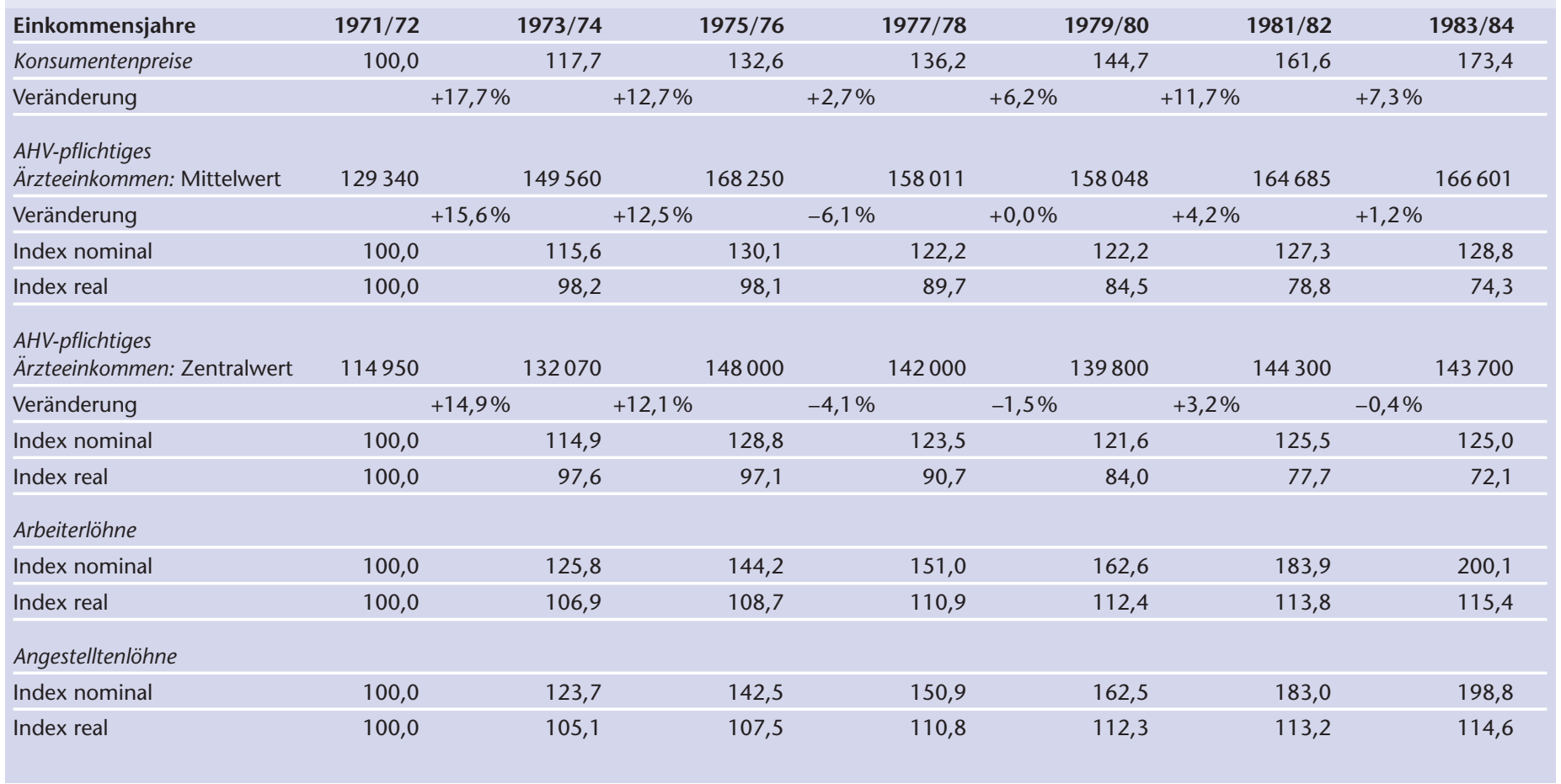

\section{Tabelle 23b}

Indexzahlen des AHV-pflichtigen Einkommens der freien Ärzteschaft der Schweiz (bis 1975/76 unter 63 Jahren, ab 1977/78 unter 66 Jahren). 1971/72 bis 2002: Zweiter Teil 1983/84 bis 1995/96. Alle Indexreihen sind auf der Basis 1971/72=100,0 berechnet.

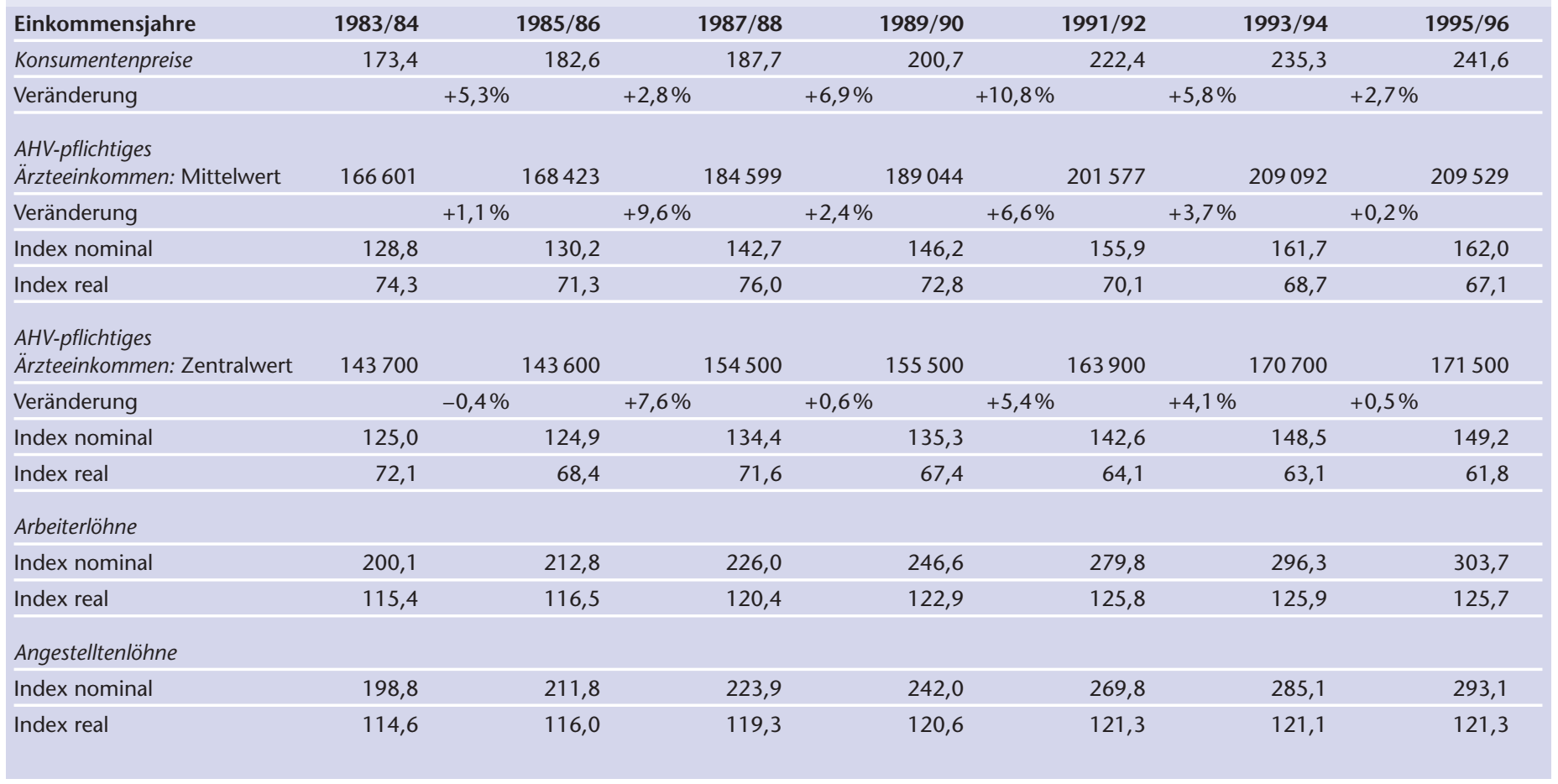




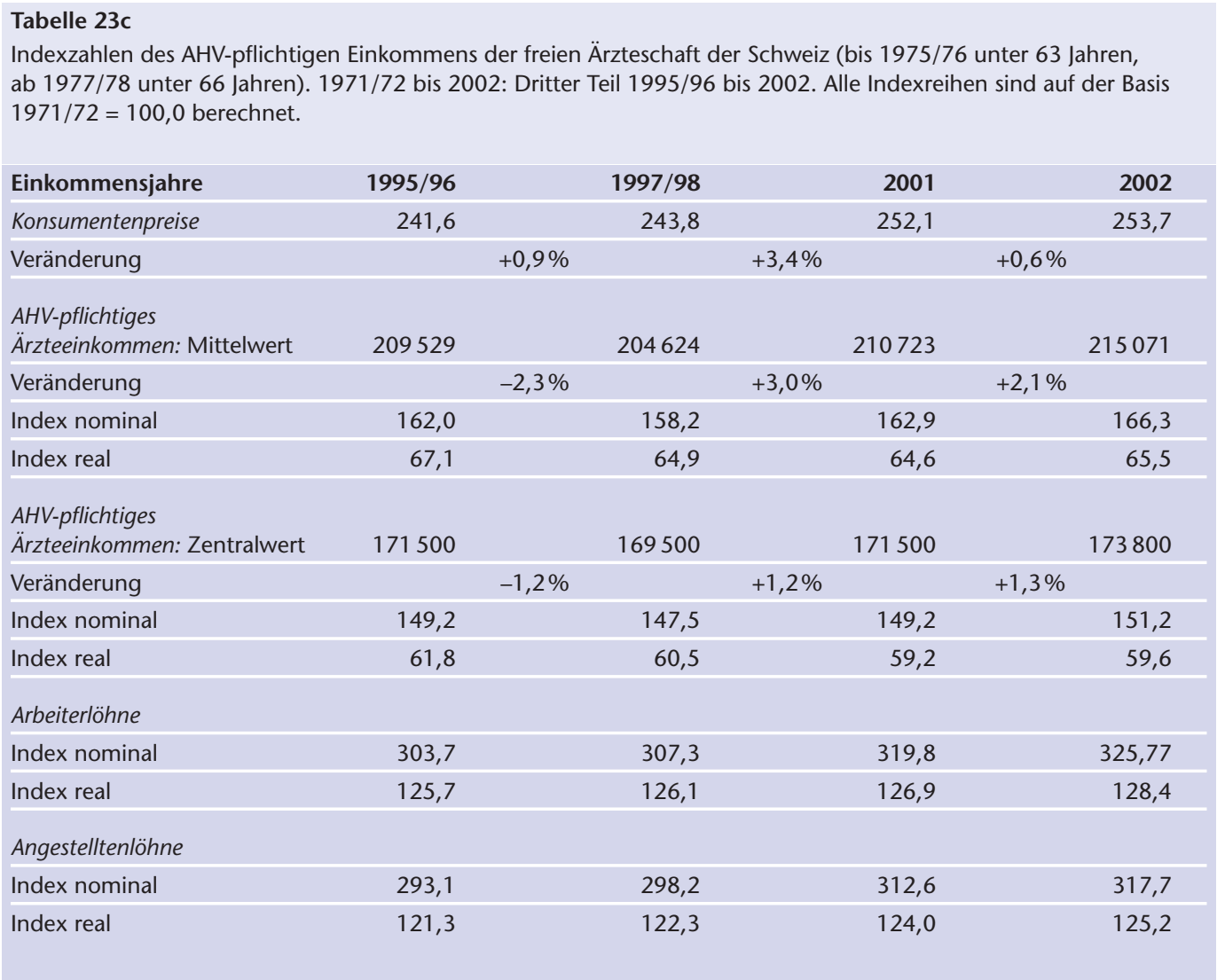

bis zwei Jahren erneut analysiert werden können und sich dabei um rund 2 bis 3 Prozent tiefere Werte ergeben, wird sich die teuerungsneutrale Entwicklung und der geringe reale Zuwachs mit Sicherheit in einen realen Einkommensverlust verwandeln.

Einkommensentwicklung 1971/72 bis 2002 Mit den revidierten Einkommenszahlen 2001 und den neuen Einkommenszahlen 2002 kann nun die Entwicklung des mittleren und des zentralen AHV-pflichtigen Einkommens der freien Ärzteschaft der Schweiz über gut drei Jahrzehnte verfolgt werden.

In den Graphiken 13 bis 16 (Anhang Seiten 52/53) sind die Indexreihen für das mittlere und das zentrale AHV-pflichtige Ärzteeinkommen (nur Ärzte unter 66 Jahren) sowohl nominal wie auch real (d. h. unter Berücksichtigung der Teuerung) dargestellt.

Von den vielen Tabellen und Graphiken im Originalgutachten (einsehbar im Internet auf der Homepage der FMH www.fmh.ch) werden hier als Beispiele die Graphiken 13 und 14, Entwicklung des Mittelwertes des AHV-pflichtigen Berufseinkommens der freien Ärzteschaft der Schweiz unter 66 Jahren nominal und real (unter Berücksichtigung der Teuerung), und die Tabellen 5 und 9 (Durchschnitt des AHV-pflichtigen
Einkommens 2001 und 2002 der in freier Praxis tätigen unter 66 Jahre alten Ärzte nach Kantonen und nach Spezialitäten) gezeigt.

\section{F.8. Gesamtaufwand der Volkswirtschaft für die freie Ärzteschaft}

Es ist schwierig, den Gesamtaufwand der Volkswirtschaft für die freie Ärzteschaft in der Schweiz zu erheben, da verschiedene Berechnungsfaktoren wie um Beispiel der Unkostensatz der Arztpraxis nur mit recht deutlicher Unsicherheit erhoben werden können.

Für die Jahre 2001 und 2002 liegt der volkswirtschaftliche Gesamtaufwand für die freie Ärzteschaft der Schweiz um die 10,016 und 10,615 Mia, was 2,37 bzw. 2,46 Prozent des Bruttoinlandproduktes BIP der Schweiz ausmacht.

Wenn wir von den Zahlen der Interpharma ausgehen, entsprechen die 10,016 Mia im Jahr 2001 21,71\% der gesamten Ausgaben für das Gesundheitswesen in der Schweiz. Für das Jahr 2002 entsprechen die 10,615 Mia 22,12\% der gesamten Ausgaben für das Gesundheitswesen in der Schweiz.

Nach Angaben der Pharma Information benötigten die Spitäler im Jahr 2001 für die stationäre und ambulante Betreuung 24,236 Mia oder $52,54 \%$ des Gesamtaufwandes für das Gesundheitswesen. 
Gemäss Angaben der Pharma Information betragen die Gesamtaufwendungen für die freie Ärzteschaft (inkl. selbstdispensierte Medikamente) im Jahr 2001 7,972 Mia oder 17,28\% des Gesamtaufwandes für das Gesundheitswesen.

Die Totaleinkommen aller Ärzte in freier Praxis belaufen sich dabei auf 3,008 Mia oder 6,52\% des Gesamtaufwandes für das Gesundheitswesen.

Nach Angaben der Pharma Information ${ }^{16}$ ) bis 22) benötigten die Spitäler im Jahre 2001 für die stationäre und ambulante Betreuung 25,644 Mia oder 53,45\% des Gesamtaufwandes für das Gesundheitswesen.

Gemäss Angaben der Pharma Information betragen die Gesamtaufwendungen für die freie Ärzteschaft (inkl. selbstdispensierte Medikamente) im Jahr 2002 8,102 Mia oder 16,89\% des Gesamtaufwandes für das Gesundheitswesen.

Die Totaleinkommen aller Ärzte in freier Praxis belaufen sich dabei auf 3,003 Mia oder 6,26\% des Gesamtaufwandes für das Gesundheitswesen.

\section{F.9. Tendenzen}

Die Ärzteeinkommen haben sich im Zeitraum 2001 bis 2002 praktisch im Gleichschritt mit der Steigerung des Lebenskostenindexes entwickelt. Es ist keine zusätzliche Steigerung der Ärzteeinkommen festzustellen.

Ganz generell kann festgestellt werden, dass in den letzten Jahren die Einkommen der operativ tätigen Ärzte wieder etwas rascher angestiegen sind als diejenigen der nicht-operativ tätigen Ärzte (= Grundversorger).

Es wird in den nächsten zwei bis vier Jahren interessant sein, die Auswirkungen des TarMedTarifs zu beurteilen, der ja auch zum Ziel hat, die operativ-technischen Leistungen zugunsten der ärztlich-intellektuellen Leistungen abzuwerten.

Die Gegenwartsbesteuerung erlaubt nun, die Einkommensverhältnisse der freien Ärzteschaft der Schweiz in jährlichem und nicht nur in zweijährigem Rhythmus zu analysieren.

Tabelle 5

AHV-pflichtiges Einkommen aller in freier Praxis tätigen Ärzte, Durchschnitte 2001 und 2002 nach Kantonen zusammen mit einem Index (Mittelwert ganze Schweiz = 100,0\%), Ärzte unter 66 Jahren.

\begin{tabular}{|c|c|c|c|c|c|c|c|}
\hline Kanton & Anzahl & 2001 & Index & $+/-$ & Anzahl & 2002 & Index \\
\hline $\mathrm{ZH}$ & 2396 & 225092 & $106,8 \%$ & $-0,8 \%$ & 2227 & 223248 & $103,8 \%$ \\
\hline$B E$ & 1727 & 204008 & $96,8 \%$ & $+8,4 \%$ & 1550 & 221106 & $102,8 \%$ \\
\hline LU & 415 & 229073 & $108,7 \%$ & $+2,1 \%$ & 384 & 233867 & $108,7 \%$ \\
\hline UR & 26 & 203565 & $96,6 \%$ & $-8,1 \%$ & 26 & 187142 & $87,0 \%$ \\
\hline SZ & 83 & 257363 & $122,1 \%$ & $-12,0 \%$ & 75 & 226608 & $105,4 \%$ \\
\hline UW & 74 & 210765 & $100,0 \%$ & $+1,6 \%$ & 68 & 214224 & $99,6 \%$ \\
\hline GL & 40 & 304498 & $144,5 \%$ & $-8,0 \%$ & 39 & 280213 & $130,3 \%$ \\
\hline ZG & 138 & 229936 & $109,1 \%$ & $+2,5 \%$ & 141 & 235740 & $109,6 \%$ \\
\hline FR & 321 & 206695 & $98,1 \%$ & $+10,2 \%$ & 319 & 227791 & $105,9 \%$ \\
\hline SO & 355 & 210676 & $100,0 \%$ & $+1,1 \%$ & 350 & 212984 & $99,0 \%$ \\
\hline BS & 446 & 170012 & $80,7 \%$ & $+3,7 \%$ & 386 & 176348 & $82,0 \%$ \\
\hline$B L$ & 471 & 236004 & $112,0 \%$ & $+2,6 \%$ & 474 & 242112 & $112,6 \%$ \\
\hline $\mathrm{SH}$ & 123 & 198933 & $94,4 \%$ & $+0,3 \%$ & 124 & 199583 & $92,8 \%$ \\
\hline AP & 97 & 212124 & $100,7 \%$ & $-0,9 \%$ & 88 & 210319 & $97,8 \%$ \\
\hline SG & 502 & 237025 & $112,5 \%$ & $-6,5 \%$ & 480 & 221620 & $103,0 \%$ \\
\hline GR & 275 & 205088 & $97,3 \%$ & $-2,5 \%$ & 243 & 199872 & $92,9 \%$ \\
\hline AG & 598 & 202233 & $96,0 \%$ & $+0,6 \%$ & 482 & 203455 & $94,6 \%$ \\
\hline TG & 276 & 238948 & $113,4 \%$ & $-2,1 \%$ & 277 & 233993 & $108,8 \%$ \\
\hline TI & 372 & 241260 & $114,5 \%$ & $+4,3 \%$ & 369 & 251712 & $117,0 \%$ \\
\hline VD & 946 & 195247 & $92,7 \%$ & $+9,1 \%$ & 551 & 212952 & $99,0 \%$ \\
\hline VS & 359 & 161247 & $76,5 \%$ & $+9,4 \%$ & 323 & 176448 & $82,0 \%$ \\
\hline NE & 164 & 143656 & $68,2 \%$ & $+0,5 \%$ & 155 & 144313 & $67,1 \%$ \\
\hline GE & 183 & 163734 & $77,7 \%$ & $+10,6 \%$ & 150 & 181056 & $84,2 \%$ \\
\hline JU & 78 & 141574 & $67,2 \%$ & $+4,6 \%$ & 75 & 148044 & $68,8 \%$ \\
\hline Schweiz & 10465 & 210723 & $100,0 \%$ & $+2,1 \%$ & 9356 & 215071 & $100,0 \%$ \\
\hline
\end{tabular}




\begin{tabular}{|c|c|c|c|c|c|c|c|}
\hline Spezialität & Anzahl & 2001 & Index & $+/-$ & Anzahl & 2002 & Index \\
\hline Anästhesie & 280 & 276861 & $131,4 \%$ & $-2,6 \%$ & 237 & 269546 & $125,3 \%$ \\
\hline Chirurgie & 505 & 259991 & $123,4 \%$ & $+5,5 \%$ & 445 & 274193 & $127,5 \%$ \\
\hline Dermatologie & 202 & 237186 & $112,6 \%$ & $+10,1 \%$ & 178 & 261041 & $121,4 \%$ \\
\hline Gynäkologie & 648 & 249477 & $118,4 \%$ & $+1,3 \%$ & 590 & 252635 & $117,5 \%$ \\
\hline Innere Medizin & 2054 & 217715 & $103,3 \%$ & $+1,6 \%$ & 1802 & 221116 & $102,8 \%$ \\
\hline Neurochirurgie & 22 & 309655 & $146,9 \%$ & $+18,3 \%$ & 23 & 366239 & $170,3 \%$ \\
\hline Neurologie & 134 & 185772 & $88,2 \%$ & $+3,1 \%$ & 120 & 191517 & $89,0 \%$ \\
\hline Ophthalmologie & 365 & 358039 & $169,9 \%$ & $+4,8 \%$ & 334 & 375067 & $174,4 \%$ \\
\hline Orthopädie & 247 & 284089 & $134,8 \%$ & $+6,4 \%$ & 230 & 302140 & $140,5 \%$ \\
\hline ORL & 187 & 286283 & $135,9 \%$ & $+6,4 \%$ & 166 & 304519 & $141,6 \%$ \\
\hline Pädiatrie & 511 & 170257 & $80,8 \%$ & $-1,7 \%$ & 464 & 167414 & $77,8 \%$ \\
\hline Physikalische Medizin & 156 & 214994 & $102,0 \%$ & $+3,0 \%$ & 139 & 221448 & $103,0 \%$ \\
\hline Psychiatrie & 1265 & 117513 & $55,8 \%$ & $+1,0 \%$ & 1125 & 118679 & $55,2 \%$ \\
\hline Kinderpsychiatrie & 192 & 102134 & $48,5 \%$ & $+1,9 \%$ & 173 & 104089 & $48,4 \%$ \\
\hline Radiologie & 127 & 391257 & $185,7 \%$ & $+8,6 \%$ & 111 & 424752 & $197,5 \%$ \\
\hline Urologie & 98 & 312410 & $148,3 \%$ & $+7,8 \%$ & 86 & 336784 & $156,6 \%$ \\
\hline Allgemeinmedizin & 3460 & 194738 & $92,4 \%$ & $+0,0 \%$ & 3122 & 194787 & $90,6 \%$ \\
\hline Spezialisten & 7005 & 218618 & $103,7 \%$ & $+3,0 \%$ & 6234 & 225230 & $104,7 \%$ \\
\hline Allgemeinmedizin & 3460 & 194738 & $92,4 \%$ & $+0,0 \%$ & 3122 & 194787 & $90,6 \%$ \\
\hline Alle & 10465 & 210723 & $100,0 \%$ & $+2,1 \%$ & 9356 & 215071 & $100,0 \%$ \\
\hline
\end{tabular}

\section{Abbildung 13}

Entwicklung des Mittelwertes des AHV-pflichtigen Einkommens der freien Ärzteschaft der Schweiz unter 66 Jahren (nominal) $1971 / 72$ bis 2002 .

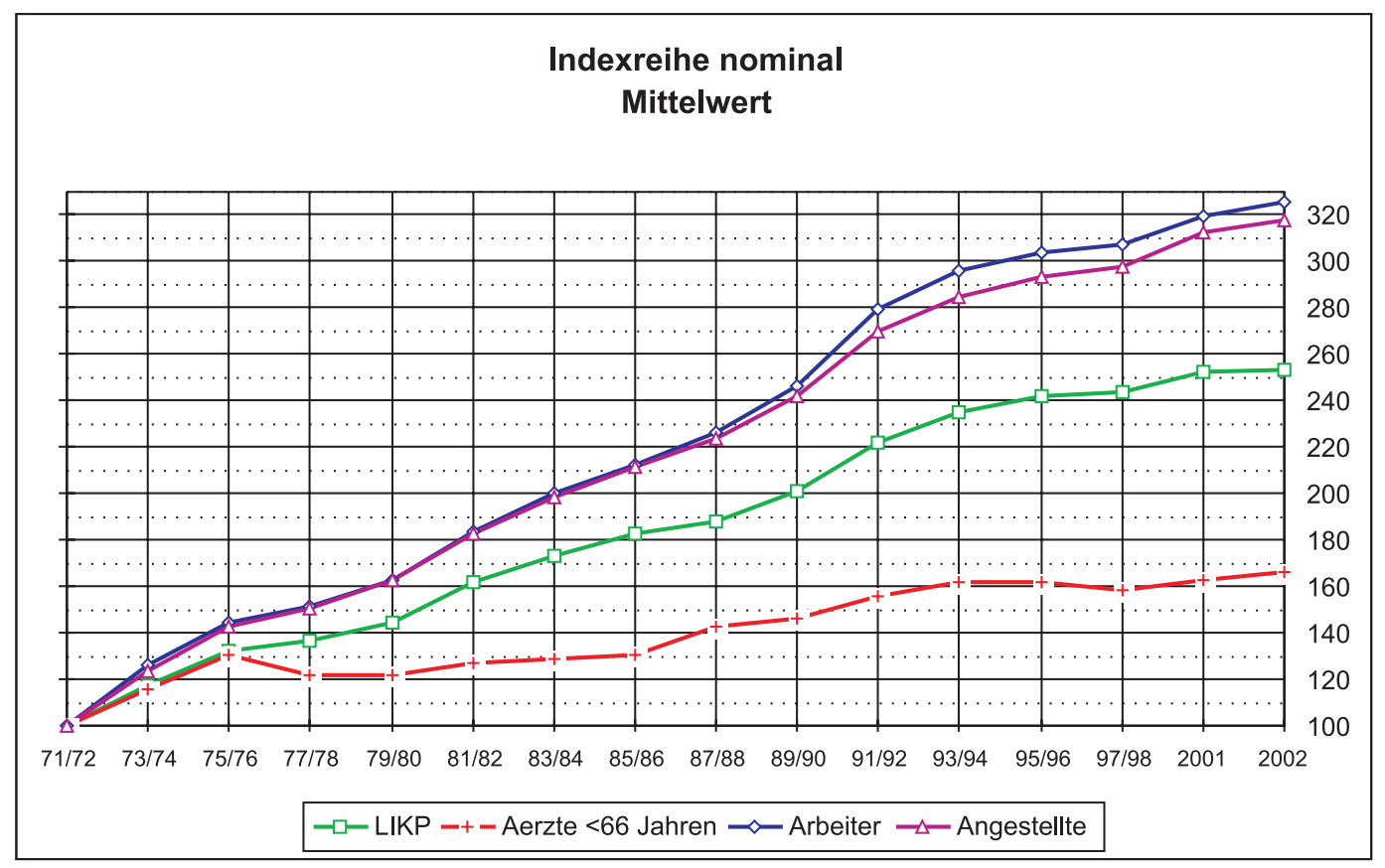


Abbildung 14

Entwicklung des Mittelwertes des AHV-pflichtigen Einkommens der freien Ärzteschaft der Schweiz unter 66 Jahren (real) $1971 / 72$ bis 2002

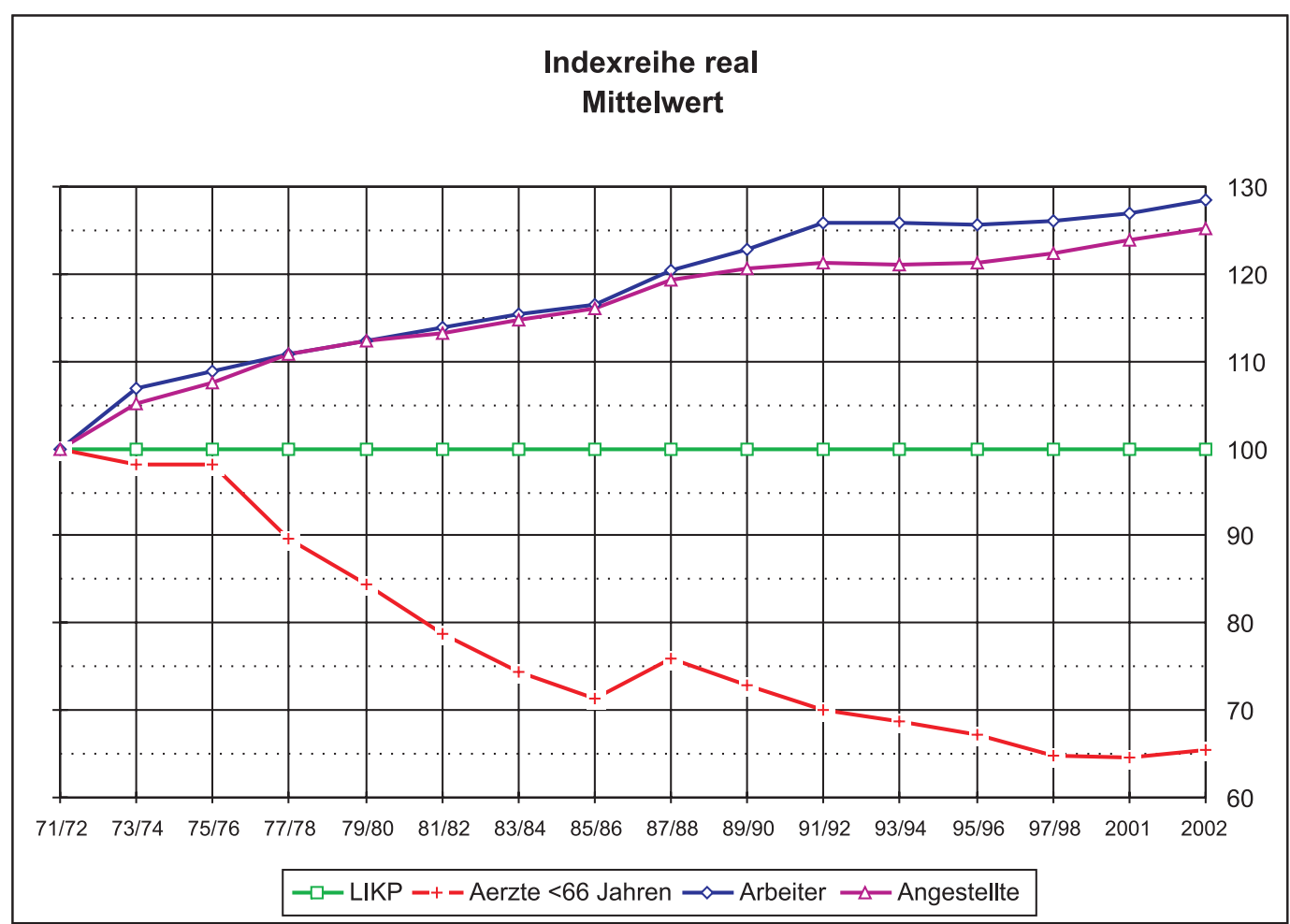

